\title{
Berimbau: a simple instrument for teaching basic concepts in the physics and psychoacoustics of music
}

\author{
Rui C. Vilão* and Santino L. S. Melo ${ }^{\dagger}$ \\ CEMDRX, Department of Physics, University of Coimbra, \\ Rua Larga, P-3004-516 Coimbra, Portugal
}

(Dated: May 1, 2014)

\begin{abstract}
We address the production of a musical tone by a very simple musical instrument of the Brazilian tradition: the berimbau-de-barriga. The simple physics of vibrations of the string and of the air mass inside the gourd are reviewed. Simple measurements, which illustrate the basic physical phenomena, are performed using a PC based Soundcard Oscilloscope. The inharmonicity of the string and the role of the gourd are discussed in the context of known results in the psychoacoustics of pitch definition.
\end{abstract}




\section{INTRODUCTION}

The berimbau is a well-known musical instrument of the Brazilian tradition. Its historical origins are not clear, but all evidence points to the fact that it developed autonomously in Brazil from previous African musical traditions brought with the slaves since the XVI ${ }^{\text {th }}$ century. This resulted into this specific Brazilian instrument known as berimbau-de-barriga or simply berimbau. ${ }^{1-3}$ The berimbau assumes particular importance in the musical accompaniment of the Brazilian martial art capoeira, which is now disseminated worldwide.

As we will discuss, this instrument is basically constituted of a vibrating string enhanced by a resonator. Its simple constitution, together with its easy construction and its relevance in both the musical and martial-arts cultural landscape, makes it a privileged tool for using in simple demonstrations of the basic principles of the underlying physics. It thus serves as a way to introduce simple physics and psychoacoustics principles to a relatively wide audience.

In this work we have performed some basic measurements of the sound emitted by a berimbau, using a PC based Soundcard Oscilloscope available online for private and noncommercial use in educational institutions. ${ }^{4}$ These measurements allow to illustrate the basic physics involved and to present these measurements in the perspective of a musical instrument, where psychoacoustics results bring a particularly helpful insight. In particular, we discuss the effect of the inharmonicity of the string and the role of the gourd (cabaça) in the definition of the musical tone of the berimbau and relate it to the ongoing research in the difficult question of the definition of pitch.

\section{BASIC COMPONENTS OF THE BERIMBAU AND THEIR FUNCTION}

Figure 1 illustrates the berimbau used in the course of this work: a typical berimbau is constituted of a steel string attached to a wooden bow about $1.2-1.5 \mathrm{~m}$ long. The bow is traditionally made of biriba (Rollinia mucosa), due to the resistance to bending of this wood. Biriba can however be replaced by other adequate wood such as bamboo or oak. ${ }^{3}$ The forced curvature of the bow provides the necessary tension to the string. A gourd is attached to the string and bow at about $20 \mathrm{~cm}$ from the bottom end, as illustrated in Fig. 1. This gourd is roughly spherical and is traditionally made of calabash, either coité (Crescentia 
cujete) or cabaça (Lagenaria siceraria), ${ }^{1}$ although coconut or even an empty metal can are sometimes used. ${ }^{3} \mathrm{~A}$ circular opening is cut on the gourd and its interior is hollow. The gourd is attached to the bow and string through a small cotton twine which crosses it in two small holes cut in the extremity opposite to the circular opening, as illustrated in Fig. 2. The cotton twine plays a role in the tuning of the berimbau, since it constitutes a fixed point of the string.

The instrument is usually hold by the left hand, while the string is struck by a wooden stick (baqueta) hold by the right hand (thumb, index and middle fingers). A basket rattle, an independent instrument from the organological point of view, is usually held as well by the right hand (ring and little fingers). Typically, the ring and middle fingers of the left hand hold the wooden bow at the level of the abdomen, while the little finger is placed under the tuning loop between the bow and the string. ${ }^{2}$ The thumb and index fingers hold a small stone or coin. Pressing the coin against the string reduces its effective length and increases its pitch by about one semitone to one tone. Timbrical diversity is also obtained by pressing the gourd against the abdomen, thus eliminating completely the main resonance of the gourd and obtaining a completely different timbre.

In this work we propose to specifically analyse the role of the inharmonicity of the string and that of the gourd in the timbre of the berimbau, taking into account known results in the physics and psychoacoustics of sound. For that, we begin by a short review of the basic aspects of the vibration of strings and of Helmholtz resonators.

\section{A. Normal modes of ideal strings}

We recall that the frequencies of the normal modes of vibration of a ideal string of length $L$, fixed at both ends, with linear mass density $\mu$ and subject to the tension $T$, follow the harmonic relation

$$
f_{n}=n f_{1}=n \frac{v}{2 L}=\frac{n}{2 L} \sqrt{\frac{T}{\mu}}
$$

where $v=\sqrt{\frac{T}{\mu}}$ is the velocity of the transverse sound waves in the string. In real strings, the stiffness slightly distorts the harmonicity. ${ }^{5}$ The description of the vibration modes of the stiff string has been thoroughly debated since the late XIX ${ }^{\text {th }}$ century (see refs. 6 and 7 for 
a review) and the following simple expression is found to provide an adequate description, ${ }^{6}$ justifying its abundant use in the corresponding literature: ${ }^{8-10}$

$$
\begin{aligned}
f_{n} & =n f_{0} \sqrt{1+B n^{2}} \\
B & =\frac{\pi^{3} Y d^{4}}{64 L^{2} T}
\end{aligned}
$$

$B$ is the inharmonicity coefficient, where $Y$ is the Young modulus of the string and $d$ is the diameter of its circular section. $f_{0}$ is the fundamental frequency of the same string without stiffness, which is thus found to be slightly smaller than $f_{1}$.

\section{B. Vibration frequencies associated to the gourd}

In order to understand the contribution of the gourd to the acoustics of the berimbau we need to model the frequencies of vibration associated to it. The vibrations of the mass of air delimited by the volume of the gourd and its opening are bound to play a leading role, although at first sight it may not be entirely evident which vibration modes are dominant in a real gourd: those of the entire column of air or those of the mass of air at the opening. These two limits are described by two different models: the pipe model for the former and the Helmholtz resonator model for the latter.

\section{Pipe model}

In the case where the vibrations of the entire column of air, with length $L$, are dominant, it is usual to distinguish whether the column is free to vibrate at both ends of the pipe or only at one end. ${ }^{5}$ In the first case, the frequencies of the vibration modes are again simply given by the purely harmonic relation

$$
f_{n}=n f_{1}=n \frac{v_{0}}{2 L}
$$

where $v_{0}$ represents the velocity of sound in air. In the case where the pipe is closed in one of the ends we have instead:

$$
f_{n}=n f_{1}=(2 n-1) \frac{v_{0}}{4 L}
$$


Of course, if any of these pipe models is to be applied to the gourd, it is likely to be the open-closed pipe model. The model of the pipe open at both ends, if applicable, would be limited to the case where the holes associated to the cotton twine were large enough to ensure that the air pressure at the corresponding end was kept always equal to the atmospheric pressure. This is not usually the case, since the cotton twine closely fits the holes of the gourd.

\section{Helmholtz resonator}

In the case of a recipient of volume $V$ with a neck of length $L$ and circular cross-section $S=\pi a^{2}, a$ being the radius of the opening, the mass of air inside the volume $V$ can be set to vibrate by the small mass of air inside the neck with a frequency given by (assuming that the wavelength is much longer than the dimensions of the resonator $)^{5,12}$

$$
f_{H}=\frac{v_{0}}{2 \pi} \sqrt{\frac{S}{V L}}
$$

where $v_{0}$ is the velocity of sound in air. This acoustical device is usually known as Helmholtz resonator. In the case of the berimbau, the gourd presents no neck. However, an effective length of the neck can be estimated by using twice the end correction of a tube of radius $a .{ }^{5}$ For the case of a flanged tube, Lord Rayleig estimated the end correction $c$ to $\mathrm{be}^{13}$

$$
\frac{\pi}{4} a<c<\frac{8}{3 \pi} a
$$

The corresponding effective length $L_{\text {eff }}=2 c$ corresponds to $1.5708 a<L_{\text {eff }}<1.6977 a$. For the more realistic case of an unflanged circular pipe, Levine and Schwinger found the end correction to decrease as a function of frequency and to be $c=0.6133 a$ for the long wavelength (low frequency) limit. ${ }^{14}$ The corresponding low-frequency effective length of the neck thus results to be

$$
L_{e f f}=1.2266 a
$$

A refined analysis of the Helmholtz resonator is presented by Fletcher and Rossing, ${ }^{5}$ where the frequencies of the higher vibration modes are obtained assuming that the neck is 
narrow enough so that the diameter of the cavity is kept well below the wavelength. Fletcher and Rossing find the resonance frequencies to obey the relation:

$$
\tan \left(\frac{2 \pi L f}{v_{0}}\right)=\frac{S v_{0}}{2 \pi V} \frac{1}{f}
$$

Equation 8 of course reduces to eq. 5 in the limit where $\lambda=v_{0} / f>>L$. It is also possible to show that this refined model contains the pipe model as a limit, both for the case where the pipe is open at both ends and for the case where it is open at one end only. The latter case corresponds to the limit of large volume $V$ of the cavity, where equation 8 can be simplified to

$$
\frac{2 \pi L f}{v_{0}} \sim n \pi \rightarrow f \sim n \frac{v_{0}}{2 L}
$$

In the case of small volume of the cavity, equation 8 reduces to

$$
\frac{2 \pi L f}{v_{0}} \sim \frac{\pi}{2}+n \pi \rightarrow f \sim \frac{v_{0}}{4 L}+n \frac{v_{0}}{2 L}
$$

With respect to the modelling of the gourd, we note that the length to be considered in equation 9 is that of the neck only. In the case of the neckless gourd used here, it corresponds

to $L_{e f f}=1.2266 a$ (eq. 7), using the model of Levine and Schwinger. ${ }^{14}$ The limit expressed by eq. 10 corresponds to the pipe model of equation 4 using the length of the gourd as the length of the pipe.

\section{EXPERIMENTAL RESULTS AND DISCUSSION}

\section{A. Experimental Details}

We adopted a very simple experimental setup which is easily available to any class-room where a computer is present. We investigated the sound spectrum of the berimbau using a microphone connected to a laptop, analysed through a PC based Soundcard Oscilloscope accessible online for private and non-commercial use in educational institutions. ${ }^{4}$ This software performs basic measurements and analysis of sound. We have used its fast-Fourier transform (FFT) capabilities in order to obtain the frequency spectrum of our instrument and of its parts. 
In order to reduce background noise, the measurements were realized in an inner quiet room. A Sony ECM-DS70P microphone was used, with a response bandwidth in the range $100 \mathrm{~Hz}-15 \mathrm{kHz}$. The microphone was placed at about $0.5 \mathrm{~m}$ from the instrument in a direction perpendicular to its main plane. Room temperature was about 22(1) degrees Celsius, so that we expect the corresponding sound velocity in air to be $344(1) \mathrm{m} / \mathrm{s}$, using the known expression ${ }^{5}$

$$
v_{\text {sound }}=332\left[1+0.00166 \mathrm{~T}\left({ }^{\circ} \mathrm{C}\right)\right] \mathrm{m} / \mathrm{s}
$$

In order to compute the fast Fourier transform, a time record of 0.12512 seconds was used, sampled in $N=5512$ intervals of $\Delta=2.27 \times 10^{-5}$ seconds. The sampling rate is $f_{s}=1 / \Delta=44.1 \mathrm{kHz}$ and the resolution is estimated to be $\sigma_{f} \sim f_{s} / N=8 \mathrm{~Hz}$. No window function was used.

The string used in this work was a common steel string with a radius $r=0.5(1) \times 10^{-3} \mathrm{~m}$. The effective vibrating length was $L_{p}=1.105(5) \mathrm{m}$ with the gourd in place and $L_{r}=1.370(5) \mathrm{m}$ with the gourd removed. The Young modulus of steel is $^{15} Y=200 \times 10^{9} \mathrm{~Pa}$ and its density is ${ }^{15} \rho=7.8 \times 10^{3} \mathrm{~kg} \mathrm{~m}^{-3}$. We thus estimate the linear density of our string to be $\mu=\pi r^{2} \rho=6(2) \times 10^{-3} \mathrm{~kg} \mathrm{~m}^{-1}$. The gourd used in this work has an interior volume of $0.90(5) \times 10^{-3} \mathrm{~m}^{3}$. The diameter of its circular opening is $7.3(1) \times 10^{-2} \mathrm{~m}$ and its length (from the opening to the cotton twine) is $13.5(1) \times 10^{-2} \mathrm{~m}$.

\section{B. String with the gourd removed}

\section{Experimental data and analysis}

We began by investigating the sound spectrum of the bare string with the gourd removed. The string was plucked with the finger at about $13 \mathrm{~cm}$ from the bottom end. We chose to pluck the string instead of striking it with the stick because this provides stronger spectral power, thus easier to detect. We note that the plucking point (or the striking point when the berimbau is properly played) has some influence on the spectral weight: the vibration modes presenting a node at that point are known to be suppressed. ${ }^{5}$ For example, if the string is hit at a fraction $1 / 10$ of its length, the $10^{\text {th }}$ vibration mode and its multiples $\left(20^{\text {th }}\right.$, $30^{\text {th }}$, etc) are likely to be suppressed. 
In Fig. 3 we show the Fast Fourier Transform (FFT) spectrum as obtained from the Soundcard Oscilloscope program, for the berimbau of Fig. 1. The central frequencies corresponding to the first 28 modes are represented in Table I. The fundamental vibration of the string is found at about $f_{1}=100(5) \mathrm{Hz}$. In Table I we also show the interval in cents $\left(\Phi_{\exp }\right)$ with respect to the ideal harmonic relation based on the same $100 \mathrm{~Hz}$ fundamental frequency. We recall that the ratio $I=f_{n} /\left(n f_{1}\right)$ of the frequency $f_{n}$ of the $n^{\text {th }}$ mode and the frequency $n f_{1}$ expected from a purely harmonic relation can be translated to the more convenient cent $(\$)$ scale for musical intervals. ${ }^{16,17} 1200$ cents represent a frequency ratio of 2 , matching the important relation of one octave, so that the number of cents associated to a specific frequency ratio $I$ is

$$
n=1200 \frac{\log _{10}(I)}{\log _{10}(2)}
$$

Two aspects are clearly visible in figure 3 :

1. The lowest modes $\left(3^{\text {rd }}\right.$ to $\left.7^{\text {th }}\right)$ are underrepresented in the spectrum.

2. The modes above the $10^{\text {th }}$ have a very important spectral weight in the region $1-3$ $\mathrm{kHz}$, where the frequency detection of the ear is most accurate. ${ }^{16,18}$

We will address the implications of these two features in detail below. However, we add two important additional notes on the FFT spectrum shown in figure 3:

- we note that the $8^{\text {th }}$ mode is particularly prominent in the spectrum. The selective enhancement of this particular mode may arise from a modal frequency of the stick itself. The modal frequencies of the stick can in principle be measured by damping the string and replacing the gourd with an acoustically dead object with the same mass, while keeping equal the tension of the string. However, with our present equipment the amplitudes of vibration of the modal frequencies of the stick are comparable to background. We will therefore not pursue this particular feature, that does not affect our overall conclusions.

- the conspicuous absence of the $10^{\text {th }}$ and $20^{\text {th }}$ modes are consistent with having the string plucked at about $1 / 10$ of its length, as discussed above. 
With respect to the two main features mentioned above, we will first address the implications of the high spectral weight in the region $1-3 \mathrm{kHz}$ and come back to the low spectral weight of the lowest modes in section IIID. The strong spectral weight of the highest modes is responsible for the characteristic non-consonant metallic timbre of the string. In order to develop this point in a more quantitative way, we first note that the ratio $I$ of the frequency $f_{n}$ of the actual $\mathrm{n}^{\text {th }}$ harmonic to the frequency $n f_{1}$ expected from the harmonic relation is, from eq. 2:

$$
I=\frac{f_{n}}{n f_{1}}=\sqrt{1+B n^{2}}
$$

where $f_{1}$ is the fundamental frequency of the stiff string. In order to analyse this aspect in more detail, we represent in Fig. 4 an expanded view of Fig. 3 in the region $1-3 \mathrm{kHz}$, where the spectral power is particularly relevant. We will henceforth limit our analysis to frequencies up to $3 \mathrm{kHz}$.

In Fig. 5 we represent the harmonics assigned in Fig. 4 as a function of the harmonic number $n$. The full line is a fit to Eq. 2, yielding $f_{0}=99.5(1) \mathrm{Hz}$ and $B=9.7(3) \times 10^{-5}$. This value for $f_{0}$, together with Eq. 1 and the experimental values quoted in section III A, allows to use Eq. 2 to predict $B=12(4) \times 10^{-5}$, which is consistent with the fitted value. The dashed line represents the harmonic relation $f_{n}=n f_{1}$, which is clearly not followed for $n>20$. In Table I we see that the relation of the vibration frequency to that expected from the ideal harmonic relation, for $n>20$, ranges from $20 \phi$ to 59 , thus up to about a quarter of tone. This is above the differential threshold of perception in the spectral region $1-3 \mathrm{kHz},{ }^{16,18}$ suggesting that the inharmonicity of the steel string of the berimbau may be perceptible.

\section{Inharmonicity and pitch perception}

The relation of the inharmonicity of strings to the perception of pitch has been investigated for a long time. Fletcher et al. have suggested that the inharmonicity of the strings of the first three octaves of the piano is essential for its liveness or warmth. ${ }^{8}$ Pure harmonicity is not essential for pitch perception ${ }^{19}$ and the concept of harmonic sieve has been proposed in this context: spectral components are perceptually grouped if their frequencies fall within a certain range around integer multiples of a common fundamental frequency $f_{0} \cdot{ }^{19,20}$ All this 
of course implies relatively small values of inharmonicity. Järveläinen et al. have measured the audibility threshold of inharmonicity as a function of the fundamental frequency $f_{0}$ and verified that it correlates with the inharmonicity coefficient B according to the following expression: ${ }^{9}$

$$
\ln B=2.57 \ln f_{0}-26.5
$$

For $f_{0}=100 \mathrm{~Hz}$, this model predicts that the inharmonicity is clearly perceived if $\mathrm{B}$ is larger than $4.3 \times 10^{-7}$. Our fitted value $B=9.7(3) \times 10^{-5}$ is about 200 times larger.

Chin and Berger ${ }^{10}$ review recent results regarding inharmonicity and pitch perception and state that "a moderate amount of inharmonicity creates a sense of warmth, but an excessive amount of inharmonicity can result in a harsher metallic sound." They find that the perception of inharmonicity is highly dependent on the individual musical background and training and suggest that the threshold for perception of inharmonicity can be mapped out for each individual (and that, of course, it can change for a given individual with musical training). Their results also suggest that for such a low fundamental frequency $f_{0}=100 \mathrm{~Hz}$ as that of our string, an inharmonicity coefficient as high as our fitted value $B=9.7(3) \times 10^{-5}$ is likely to be well above the audibility threshold even for the more insensitive individuals. We thus conclude that the high inharmonicity coefficient of the steel string of the berimbau is likely to be responsible for the harsh metallic sound of the bare steel string.

Most importantly, the near absence of the lowest modes brings additional difficulties for the perception of the pitch of the string tone, as will be discussed below.

On a side note, we recall that the berimbau player uses a coin/stone in order to reduce the effective length of the string and concomitantly increase its fundamental frequency of vibration. This increase is known to amount from about one semitone to one tone. ${ }^{3}$ We note that the length reduction is of course dependent on the size of the hand of the player and the inclination of the stone/coin with respect to the string, but we may estimate a reduction of $5-15 \mathrm{~cm}$ to be a typical value. In the case of our string (effective length $L=1.105 \mathrm{~m}$ ), this corresponds to a length reduction of about $4-14 \%$ and to an increase of the fundamental frequency in the range $70-230 \propto$. Notwithstanding the fact that these simple estimates disregard the possible effect of the change in the tension of the string due to the use of the coin/stone, they are consistent with the known increase of the pitch in the one semitone to one tone range. 


\section{Gourd}

We have also measured the sound spectrum due to the gourd alone, by tapping with a soft mallet the end of the gourd with the twine. The corresponding FFT spectrum is shown in Fig. 6, limited as before to $3 \mathrm{kHz}$ for clarity. A similar spectrum is obtained by gently blowing at the opening of the gourd. We observe two resonances which we can adequately fit to a Gaussian shape $A \exp \left(-\frac{1}{2}\left(\left(f-f_{g}\right) / \sigma\right)^{2}\right)$, obtaining $f_{g 1}=555(20) \mathrm{Hz}$ and $f_{g 2}=2111(15) \mathrm{Hz}$ (the numbers in parenthesis denote the respective fitted values $\sigma$ ).

We note that eq. 5 predicts the fundamental vibration frequency of the gourd to be $f_{g 1}=558(17) \mathrm{Hz}$, when considering the realistic value $L_{e f f}=2 \times 0.6133 a=4.48 \mathrm{~cm}$ for the effective length of the neck. This value is consistent with the experimental frequency of the first mode of vibration of the gourd. In contrast, the quarter wavelength pipe model (eq. 4) predicts $f_{g 1}=637(5) \mathrm{Hz}$. The Helmholtz resonator model thus seems adequate in order to describe the first vibration model of the gourd.

We may use Eq. 8 to predict the frequency $f_{g 2}$ of the second vibration mode. Eq. 8 can be solved either graphically, iteratively or analytically using the small-angle approximation $\tan (x+n \pi) \sim x$. With this approximation we recover eq. 5 for $n=0$; for $n>1$ the following expression can be used to estimate the frequencies $f_{g n}$ of the vibration modes:

$$
f_{g n} \sim n \frac{v_{0}}{4 L_{e f f}}\left(1+\sqrt{1+\frac{4 L_{e f f} S}{n^{2} \pi^{2} V}}\right)
$$

We present in fig. 7 a graphical solution using the geometrical parameters of the gourd used in this work (including $L_{e f f}=2 \times 0.6133 a=4.48 \mathrm{~cm}$ ). The small-angle approximations are represented in Fig. 7 as dashed lines. From Fig. 7, a refined graphical value for the frequency of the first mode $f_{g 1}=540(17) \mathrm{Hz}$ is obtained, as well as the frequency of the second mode $f_{g 2}=3.92(4) \mathrm{kHz}$.

The predicted frequency of the second mode is thus almost the double of the experimentally observed value, implying that the Helmholtz resonator model fails to describe this vibration mode. In fact, the wavelength corresponding to the second resonance $f_{g 2}$ is only about $16 \mathrm{~cm}$, which is comparable to the dimentions of the resonator. This implies that it is no longer valid the assumption that the mass of air within the (effective) neck vibrates coherently while the mass of air inside the volume of the resonator is kept at uniform pressure. We thus expect that a larger mass of air is now set in vibration, so that an intermediate 
model between the Helmholtz resonator and the quarter-wavelength (open-closed) pipe is needed. In fact, the quarter wavelength (open-closed) pipe-model (eqs. 4 and 10) predicts $f_{g 2}=1911(40) \mathrm{Hz}$ for the frequency of the second vibration mode (using the full length of the gourd $L=0.135 \mathrm{~m}$ ). This provides a far better estimate than the simple Helmholtz model.

We note that the large diversity of gourd geometries used in the building of berimbaus ${ }^{21}$ is likely to provide examples covering both the pure Helmholtz resonator model or the pure quarter wavelength (open-closed) pipe model, as well as cases such as that of our gourd where neither model provides a fully adequate description.

\section{The berimbau as it is played}

\section{Experimental data and analysis}

We then proceeded to investigate the FFT spectrum of the berimbau with the gourd in place. The reduced length of the string was $1.105(5) \mathrm{m}$. We have investigated the two main playing configurations:

(a) with the gourd pressed against the abdomen of the player;

(b) with the gourd free to vibrate.

The stick has been used to excite the string in both cases. We have hit the string close to the bottom end (always less than $10 \mathrm{~cm}$ from the cotton twine): this was adopted in order to avoid for the lower harmonics the already mentioned effect of suppression of the vibration modes presenting a node at the hitting point of the stick. ${ }^{5}$ We have hit the string always at a fraction of its length smaller than $1 / 11$, implying that the modes affected by this suppression effect are higher than the $11^{\text {th }}$.

The FFT spectra corresponding to both cases (a) and (b) mentioned above are shown in Fig. 8 (a) and (b), respectively. We note that the spectral power is now much reduced with respect to that observed in section IIIB, particularly for the lower harmonics in case (a). The fundamental mode of vibration is not observed in both cases. This low spectral power of the string hit with the stick was the main reason, as mentioned already, for our option of plucking the string with the finger in section III B. The vertical scale of Fig. 8 (a) was 
thus multiplied by a factor 4 with respect to that of Fig. 8 (b), in order to make its details perceptible.

The most important effect visible in Fig. 8 is the clear enhancement, for the case (b) where the gourd is left free to vibrate, of the string harmonics around the fundamental resonance of the gourd $\left(f_{g 1}=555(20) \mathrm{Hz}\right)$, in particular the immediately adjacent $5^{\text {th }}$ vibration mode. The central frequencies for both cases (a) and (b) are listed in Table II, together with the corresponding FFT amplitudes relative to the $2^{\text {nd }}$ vibration mode for case (a).

The dashed line in Fig. 8 (b) is a Gaussian curve with center frequency $555 \mathrm{~Hz}$ and width $20 \mathrm{~Hz}$. As shown in Fig. 8(b) and represented in Table II, the presence of the gourd results in the enhancement by about one order of magnitude of the FFT relative amplitude of the $3^{\text {rd }}$ to $7^{\text {th }}$ modes, which thus assume a dominant spectral weight. A complete modelling of the gourd-string-bow system is bound to clarify the enhancement in a spectral region larger than the spectral width of the first vibration mode of the gourd. This is however outside the scope of this work, where we simply take note of this experimental result.

We will now discuss the consequences of this effect to the perception of the pitch of the berimbau with the freely vibrating gourd. We note that for the gourd pressed against the abdomen, the amplification due to the vibration modes of the gourd is simply suppressed.

\section{About the contribution of upper harmonics to the perception of the pitch of the berimbau}

As we have mentioned already, the perception of pitch is a rather complex phenomenon which goes much beyond the simple perception of the fundamental and that keeps defying full understanding. ${ }^{22-25}$ It is known for long that the perception of pitch cannot be reduced to the simple identification of the fundamental and that the presence of higher harmonics plays not only a non-negligible, but sometimes an essential role in this perception. The problematics of the perception of a pitch that may not even correspond to any frequency present in the sound spectrum is known as the problem of missing fundamental, virtual pitch or residue perception. In a seminal work, ${ }^{26}$ Plomp demonstrated that, for fundamental frequencies below $700 \mathrm{~Hz}$, the pitch of complex tones is determined by the harmonics and not by the fundamental. Moreover, his investigations clearly showed that for fundamental frequencies up to about $350 \mathrm{~Hz}$, the pitch is determined by the $4^{\text {th }}$ and higher harmonics. Subsequent studies by Ritsma, ${ }^{27}$ Bilsen, ${ }^{28}$ Houstma and Smurzynski, ${ }^{29}$ and Meddis and 
Hewitt ${ }^{30}$ have confirmed this fundamental result, allowing Meddis and Hewitt to summarize that "for fundamental frequencies below $400 \mathrm{~Hz}$, harmonics lying between the $3^{\text {rd }}$ and the $5^{\text {th }}$ contribute most to the strength of the pitch percept." ${ }^{30}$ Models for rationalizing these results include periodicity theories, place theories and processing by the central nervous system. ${ }^{25}$ However, the basic experimental psychophysical data seem to be well described by a simpler fundamental nonlinear dynamics model. ${ }^{25}$

Our measurements do show that in the usual playing configurations the fundamental vibration mode of the string is nearly absent. Moreover, the gourd is seen to contribute to a significant enhancement of the amplitude of the specific harmonics which are known to be more important for the definition of pitch. In the context of the above-mentioned results from the psychoacoustics of pitch, our measurements thus strongly suggest that the gourd is likely to play a fundamental role in the definition of pitch, through an amplification of the higher harmonics, especially the $4^{\text {th }}$ and $5^{\text {th }}$ harmonics. These have been identified as important for the perception of pitch in psychoacoustic experiments. Of course, the full development of this idea implies further thorough psychoacoustic studies that this work does not intend to provide.

\section{CONCLUSIONS}

Being a very cheap and accessible instrument, the berimbau can be easily introduced as the subject of a simple experimental study of the vibration of a (harmonic-rich) metallic string and its coupling to a narrow-filtering resonator. The widespread use of the berimbau associated to the corresponding dissemination of capoeira, makes it appealing to a particularly broad audience.

Despite its simplicity, this study allows to discuss rather complex phenomena in the physics and psychoacoustics of sound. The details of the vibration of (stiff) strings and resonators can be addressed in a simple way, but its interpretation in the musical context implies that attention must be paid to the results of ongoing research in the psychoacoustics of pitch perception. These results can thus also be illustrated.

Our measurements reveal that the stiff steel string has a strong spectral weight in the region $1-3 \mathrm{kHz}$, and that the inharmonicity of the corresponding vibration frequencies is likely to be detected even by the more insensitive individuals. The gourd is found to boost 
the vibration modes around the $5^{\text {th }}$ harmonic. These modes are known to play an essential role in the definition of pitch of complex sounds with low fundamental frequencies, as in the case of the berimbau.

The berimbau thus proves to be a useful tool that can easily be used for undergraduate classroom projects in the physics and psychoacoustics of pitch. For example, gourds with different sizes could be used in a classroom project in order to explore the effect of the geometry of the gourd discussed in sections IIB 2 and III C. Also, eq. 2 can be used to synthesize computationally a slightly inharmonic complex sound in order to individually test pitch perception. The results reviewed in sections III B 2 and III D 2 can be explored similarly, for example synthesizing complex sounds containing only the partials relevant to the perception of the missing fundamental.

\section{ACKNOWLEDGMENTS}

This work was supported from funds from FEDER (Programa Operacional Factores de Competitividade COMPETE) and from FCT - Fundação para a Ciência e Tecnologia under the project PEst-C/FIC/UI0036/2011.

* ruivilao@fis.uc.pt

$\dagger$ permanent address: Departamento de Física, Universidade Federal do Ceará, Campos do Pici, Bloco 922, Fortaleza, Ceará, Brasil

1 Kay Shaffer, O berimbau-de-barriga e seus toques [The Berimbau and Its Rhythms] (Ministério da Educação e Cultura, Rio de Janeiro, 1976).

2 Eric Galm, The berimbau: soul of brasilian music (University Press of Mississippi, Jackson, 2010).

3 Richard P. Graham and N. Scott Robinson, "Berimbau" in: Continuum Encyclopedia of Popular Music of the World, Volume 2: Performance and Production, edited by John Shepherd, David Horn, Dave Laing, Paul Oliver, and Peter Wicke (New York: Continuum, 2003), pp. 345-349 (an unedited version is also available at <http://www.nscottrobinson.com/berimbau.php>).

4 C. Zeitnitz, the Soundcard Oscilloscope program may be found at 
$<$ http://www.zeitnitz.de/Christian/scope?mid=2>.

5 Thomas D. Rossing and Neville H. Fletcher, Principles of Vibration and Sound, $2^{\text {nd }}$ edition (Springer-Verlag, New York, 2004).

6 Robert W. Young, "Inharmonicity of Plain Wire Piano Strings", Journal of the Acoustical Society of America 24, 267 - 273 (1952).

7 Harvey Fletcher, "Normal Vibration Frequencies of a Stiff Piano String", Journal of the Acoustical Society of America 36, 203 - 209 (1964).

8 Harvey Fletcher, E. Donnell Blackam, and Richard Stratton, "Quality of Piano Tones", Journal of the Acoustical Society of America 34, 749 - 761 (1962).

9 Hanna Järvelain, Vesa Välimäki and Matti Karjalainen, "Audibility of the timbral effects of inharmonicity in stringed instrument tones", Acoustics Research Letters Online 2, 79 - 84 (2001).

10 Shin Hui Lin Chin and Jonathan Berger, "Analysis of Pitch Perception of Inharmonicity in Pipa Strings Using Response Surface Methodology", Journal of New Music Research 39, 63 $73(2010)$.

11 Alejandra Kandus, Friedrich Wolfgang Gutmann, and Caio Mário Castro de Carvalho, "A física das oscilações mecânicas em instrumentos musicais: exemplo do berimbau", Revista Brasileira de Física 28, 427 - 433 (2006).

12 Joe Wolfe, Helmholtz resonance, <www.phys.unsw.edu.au/jw/Helmholtz.html>. This is a rather comprehensive webpage on the Helmholtz resonator, included in the Musical Acoustics webpages of the University of New South Wales, Australia.

13 John William Strutt, Baron Rayleigh, The Theory of Sound, Vol. II, $2^{\text {nd }}$ edition (Macmillan, London, 1929), §307 and Appendix A.

14 Harold Levine and Julian Schwinger, "On the Radiation of Sound from an Unflanged Circular Pipe", Physical Review 73, 383 - 406 (1948).

15 CRC Handbook of Chemistry and Physics, $78^{\text {th }}$ edition, edited by David R. Lide (CRC Press, Boca Raton, Florida, 2008).

16 Juan G. Roederer, Physics and Psychophysics of Music, $4^{\text {th }}$ edition (Springer-Verlag, New York, 2008).

17 Alexander J. Ellis and Alfred J. Hipkins, "Tonometrical Observations on Some Existing NonHarmonic Musical Scales", Proceedings of the Royal Society of London 37, 368-385 (1884). 
18 E. Zwicker, G. Flottorp, and S. S. Stevens, "Critical Band Width in Loudness Summation", Journal of the Acoustical Society of America 29, 548 - 557 (1957).

19 Christophe Micheyl and Andrew J. Oxenham, "Pitch harmonicity and concurrent sound segregation: Psychoacoustical and neurophysiological findings", Hearing Research 266, 36 - 51 (2010).

20 Alain de Cheveigné, "Harmonic fusion and pitch of mistuned partials", Journal of the Acoustical Society of America 102, 1083-1087 (1997).

21 Pablo Castellanos-Macín and Julius O. Smith, "Towards a physical model of the berimbau: Obtaining the modal synthesis of the cabaza", J. Acoust. Soc. America 134, 4243 (2013).

22 Florian Gomez, Victor Saase, Nikolaus Buchheim, and Ruedi Stoop, "How the Ear Tunes In to Sounds: A Physics Approach", Physical Review Applied 1, 014003 (7 pages) (2014).

23 Stefan Martignoli and Ruedi Stoop, "Local Cochlear Correlations of Perceived Pitch", Physical Review Letters 105, 048101 (4 pages) (2010).

24 Yu. V. Ushakov, A. A. Dubkov, and B. Spagnolo, "Regularity of Spike Trains and Harmony Perception in a Model of the Auditory System", Physical Review Letters 107, 108103 (4 pages) (2011).

25 Julyan H. E. Cartwright, Diego L. González, and Oreste Piro, "Nonlinear Dynamics of the Perceived Pitch of Complex Sounds", Physical Review Letters 82, 5389 - 5392 (1999).

26 R. Plomp, "Pitch of complex tones", Journal of the Acoustical Society of America 41, 1526 1533 (1967).

27 Roelof J. Ritsma, "Frequencies dominant in the perception of the pitch of complex sounds", Journal of the Acoustical Society of America 42, 191 - 198 (1967).

28 F. A. Bilsen, On the influence of the number and phase of harmonics on the perceptibility of the pitch of complex signals, Acustica 28, 60-65 (1973).

29 A. J. M. Houstma and J. Smurzynski, "Pitch identification and discrimination for complex tones with many harmonics", Journal of the Acoustical Society of America 87, $304-310$ (1990).

30 Ray Meddis and Michael J. Hewitt, "Virtual pitch and phase sensitivity of a computer model of the auditory periphery. I: Pitch identification", Journal of the Acoustical Society of America 89, 2866 - 2882 (1991). 
FIGURE CAPTIONS 
TABLE I. Experimentally observed frequencies $\mathrm{f}_{\exp }$ (up to $3 \mathrm{kHz}$ ) and intervals $\Phi_{\text {exp }}$ in cents with respect to the ideal harmonic frequency $\mathrm{f}_{\text {ideal }}=n f_{1}$, for the string with the gourd removed (as represented in Fig. 3 and in Fig. 4).

\begin{tabular}{|c|c|c|c|c|c|}
\hline $\mathrm{n}$ & $\mathrm{f}_{\exp } / 10^{2} \mathrm{~Hz}$ & $\Phi_{\exp }$ & $\mathrm{n}$ & $\mathrm{f}_{\exp } / 10^{2} \mathrm{~Hz}$ & $\Phi_{\exp }$ \\
\hline 1 & $1.00(5)$ & & 16 & $16.16(5)$ & $17(3)$ \\
\hline 2 & $2.00(5)$ & & 17 & $17.08(5)$ & $8(2)$ \\
\hline 3 & $3.00(5)$ & & 18 & $18.16(5)$ & $15(2)$ \\
\hline 4 & $4.00(5)$ & & 19 & $19.20(5)$ & $18(2)$ \\
\hline 5 & $5.00(5)$ & & 20 & $20.23(5)$ & $20(2)$ \\
\hline 6 & $6.00(5)$ & & 21 & $21.36(5)$ & $29(2)$ \\
\hline 7 & $7.00(5)$ & & 22 & $22.36(5)$ & $28(2)$ \\
\hline 8 & $8.00(5)$ & & 23 & $23.52(5)$ & $39(2)$ \\
\hline 9 & $9.00(5)$ & & 24 & $24.52(5)$ & $37(2)$ \\
\hline 10 & $10.00(5)$ & & 25 & $25.64(5)$ & $43(2)$ \\
\hline 11 & $11.00(5)$ & & 26 & $26.72(5)$ & $47(2)$ \\
\hline 12 & $12.04(5)$ & $6(3)$ & 27 & $27.84(5)$ & $53(1)$ \\
\hline 13 & $13.12(5)$ & $16(3)$ & 28 & $28.92(5)$ & $56(1)$ \\
\hline 14 & $14.09(5)$ & $11(3)$ & 29 & $30.00(5)$ & $59(1)$ \\
\hline 15 & $15.09(5)$ & $10(3)$ & & & \\
\hline
\end{tabular}

FIG. 1. The typical berimbau used in this work and its components. The full string length is $1.37 \mathrm{~m}$.

FIG. 2. Detail of the gourd. The cotton twine which attaches the gourd to the string is indicated at the left side. The diameter of the gourd opening is 7.3(1) $\mathrm{cm}$ and its inner volume was measured to be $0.90(5) \mathrm{dm}^{3}$. The length of the gourd is $13.5(1) \mathrm{cm}$ (from the opening to the cotton twine end). 
TABLE II. Central frequencies $f_{a}$ and $f_{b}$ of the first 10 harmonics of the string with the gourd in place, for the case (a) where the gourd is pressed against the abdomen and for the case (b) where it vibrates freely, respectively. The respective FFT amplitude of the given harmonic in relation to that of harmonic number 2 of case (a) is also indicated.

\begin{tabular}{ccccc}
\hline \hline Harmonic no. & $f_{a} / 10^{2} \mathrm{~Hz}$ & FFT Relative amplitude & $f_{b} / 10^{2} \mathrm{~Hz}$ & FFT Relative amplitude \\
\hline \hline 2 & $2.25(5)$ & - & - & - \\
3 & $3.32(5)$ & 1.52 & $3.33(5)$ & 4.93 \\
4 & $4.44(5)$ & 0.08 & $4.45(5)$ & 8.09 \\
5 & $5.48(5)$ & 0.71 & $5.58(5)$ & 37.16 \\
6 & $6.66(5)$ & 0.11 & $6.67(5)$ & 8.13 \\
7 & $7.80(5)$ & 0.11 & $7.83(5)$ & 7.54 \\
8 & $8.88(5)$ & 3.13 & $8.92(5)$ & 15.49 \\
9 & $10.04(5)$ & 0.92 & $10.08(5)$ & 11.14 \\
10 & $11.16(5)$ & 1.39 & $11.17(5)$ & 8.12 \\
\hline \hline
\end{tabular}

FIG. 3. Fourier transform of the vibration of the string of the berimbau, with the gourd removed. The first 9 vibration frequencies are identified. The spectral weight for harmonics above the tenth is very significant with respect to that of the lower harmonics, a characteristic feature of the metallic timbre of the string.

FIG. 4. Detailed view of Fig. 3 in the spectral region $1-3 \mathrm{kHz}$, with identification of the most prominent vibration frequencies.

FIG. 5. Frequency of the harmonics visible in Fig. 4 and presented in Table I, as a function of the harmonic number $n$. The full line is a fit to Eq. 2 and the dashed line represents the harmonic relation, as discussed in the text.

FIG. 6. Fourier transform of the vibration of the gourd, upon tapping with a soft mallet the end with the twine. Two vibration frequencies are identified at $f_{g 1}=555(20) \mathrm{Hz}$ and $f_{g 2}=2111(15)$ $\mathrm{Hz}$, as discussed in the text. 
FIG. 7. Graphical solution of eq. 8, with the parameters of the gourd used in this work (assuming $\left.L_{e f f}=2 \times 0.6133 a\right)$. The dotted lines represent $\tan (\alpha f)$, with $\alpha=2 \pi L_{e f f} f / v_{0} ;$ the dashed lines represent the corresponding small-angle approximations $\tan (\alpha f) \sim \alpha f-n \pi$; the continuous line represents $\beta / f$, with $\beta=S v_{0} / 2 \pi V$. The intersection points define the resonance frequencies $f_{g 1}=540(17) \mathrm{Hz}$ and $f_{g 2}=3.92(4) \mathrm{kHz}$.

FIG. 8. Fourier transform of the vibration of the string with the gourd in place, for the case (a) where the gourd is pressed against the abdomen and for the case (b) where it vibrates freely. The vertical scale in (a) has been multiplied by a factor 4 with respect to that in (b). A clear enhancement of the harmonics around the fundamental vibration frequency of the gourd (centered around $555 \mathrm{~Hz}$ ) is visible in (b). The dashed line in (b) is a Gaussian curve centered at $555 \mathrm{~Hz}$, as discussed in the text. 